\title{
Análisis y Reflexión sobre Modelos Teóricos del Proceso Salud-Enfermedad
}

\section{Analysis and Reflection on Theoretical Models of the Health-Disease Process}

\author{
Armando Arredondo'
}

ARREDONDO, A. Analysis and Reflection on Theoretical Models of the

Health-Disease Process. Cad. Saúde Puibl., Rio de Janeiro, 8 (3): 254-261, jul/set, 1992.

The present paper does a review and analyses the models which have been developed for the analysis of determinants and explanatory factors of the health-disease process as an elementary input for designing educational programs in the training of health manpower. Establishing the hypothesis, variables, advantages, disadvantages, time and authors of each model, the object of the analysis includes the models' methodological and/or scientific aspects: magic-religious, sanitary, unicausal, multicausal, epidemiological, ecological, social, social-historical, geographical, economic and interdisciplinary. The discussion and conclusions focus on the critical and reflexive characteristics of the models, contradictions and compatibilities among them, and their relevance for the training of human resources in health.

Keywords: Health-Disease; Determinants, Theoretical Models

\section{PLANTEAMIENTO DEL PROBLEMA}

La diversidad de modelos teóricos ó paradigmas y disciplinas involucradas en el análisis de los determinantes y condicionantes del proceso salud-enfermedad, es muy amplia, y excede a los propósitos de este artículo. Sin embargo si es un propósito fundamental mostrar un haz de luz de la diversidad y problemática operativa de propuestas de referencia disciplinaria y de la construcción del conocimiento que se ha abordado alrededor de un mismo objeto de análisis.

El pensamiento espontáneo de la totalidad, que como tendencia se mostró vigorosa en la época preburguesa y en los primeros tiempos de la burguesía, se perdió cada vez más con la atomización creciente del proceso social; en todos los ámbitos del quehacer científico se impuso la especialización de las diferentes disciplinas y la fragmentación interna del abordaje de un mismo objeto de estudio presentan-

\footnotetext{
'Instituto Nacional de Salud Pública. Av. Universidad, 655. col. Santa Maria Ahuacatitlan, Cuernavaca Mor., C.P. 62508, México.
}

dose como fenómeno concomitante la intelectualización del objeto singular aislado pagando un alto costo intelectual-social al renunciar a la realidad como un todo unitario, tanto para su abordaje teórico como operativo.

El ejercicio de la medicina está estrechamente relacionado con esta manera de abordar la realidad y con la aplicación de un conocimiento científico y tecnológico que ha sido transmitido en las aulas académicas y a través de generaciones como algo irrefutable, donde pocas veces los alumnos de medicina y/ó trabajadores de la salud se dan la tarea de hacer un análisis crítico y reflexivo sobre los diferentes modelos teóri$\cos$ contenidos en sus unidades didácticas y programas educativos que cursan o cursaron y que marcan la pauta de su práctica profesional y de la formación de futuras generaciones.

Para fines de este ensayo, se plantea abordar la problemática mencionada en el párrafo anterior tomando como objeto de análisis algunos de los modelos teóricos que desde diferentes disciplinas de estudio proponen una serie de determinantes y condicionantes del proceso salud-enfermedad. Se trata pues, de hacer una reflexión crítica sobre algunos de los modelos 
teóricos que se han desarrollado hasta la fecha y que de alguna manera han sido incluídos, inclusive determinantes, en los programas de formación de personal en salud en México y seguramente en otros países de la América Latina.

En este ensayo se partirá de la idea de que es imposible separar la salud y la enfermedad y por lo tanto su relación, por cierto muy compleja, puede describirse más como un contínuo con diferentes niveles de equilibrio que como una separación de ambos conceptos (Terris, 1975).

Dicho lo anterior, se ha visto que a través del tiempo el hombre ha elaborado diferentes modelos conceptuales sobre los determinantes del proceso salud-enfermedad. Dichos modelos han sido acordes con el nivel científico y tecnológico alcanzado y con la forma de organización social predominantes en cada época y cultura.

A pesar de que resulta aparentemente sencillo establecer cuándo y porqué una persona o un grupo ha tenido alteraciones en el proceso salud-enfermedad, y de que su equilibrio nunca ha dejado de considerarse un bien deseable por los distintos grupos sociales a lo largo de la historia, en el momento en que los profesionales de la salud deciden abandonar su experiencia cotidiana y abordan el fenómeno saludenfermedad desde todos los puntos de vista posibles se encuentran en serias dificultades.

En efecto, explicar y entender los determinantes del proceso salud-enfermedad, no es una tarea fácil ya que existen diferentes modelos para su análisis con múltiples variables, en ocasiones contrapuestas y que expresan diferencias en la manera de interpretar la realidad $y$ el mundo.

Dada su complejidad, el proceso en cuestión presenta dificultades para su comprensión y análisis. Se han desarrollado varios modelos que intentan desde diferentes perspectivas dar cuenta del fenómeno, pero hasta el momento no se ha hecho un confrontamiento de estos modelos y mucho menos un análisis cruzado tanto del conflicto de sus resultados como de las variables que cada uno considera como determinantes y de la metodología que cada autor y/ó representante siguió para llegar al modelo planteado.

Por mencionar algunas premisas que privan en el problema de estudio tomemos en cuenta que aunque, el modelo biologisista hace abstracción de los aspectos psíquicos y del entorno social, la búsqueda de indicadores objetivos que permitan la elaboración de un concepto saludenfermedad lo más cientifico posible ha obligado a los teóricos de la salud a acercarse paulatinamente al estudio de las ciencias sociales, los científicos han incorporado en sus teorías explicativas las categorias propias de la psicología, la sociología, la economía, la política, y la geografía.

Por otra parte el desarrollo del conocimiento epidemiológico ha demostrado que la determinación social de la frecuencia y distribución de los eventos patológicos se encuentra a un nivel jerárquicamente superior a la determinación biológica y psicológica de los mismos. Desde esta perspectiva, lo anterior significa que es mucho más importante la ubicación que cada individuo tiene dentro de las clases sociales, que su raza, carga genética o herencia para desarrollar nuevas patologías que en conjunto lo conducirán a la muerte.

No obstante, el desarrollo teórico-explicativo de los determinantes del proceso Salud-Enfermedad no se ha detenido; en la actualidad sigue su marcha en la búsqueda por encontrar planteamientos y enunciados científicos, objetivos $y$ operativos que expresen las leyes que rigen la determinación del proceso tanto a nivel individual como a nivel colectivo.

Luego entonces, para fines de este ensayo, la pregunta específica a contestar es la siguiente: ¿ De los modelos explicativos sobre los determinantes y condicionantes del proceso SaludEnfermedad que se han desarrollado, cuáles son sus variables de análisis y su metodología de estudio para llegar a hacer plantemientos teórico-operativos?

\section{METODOLOGIA}

A) Como primer paso metodológico para la elaboración de este ensayo, se diseñó una guía para revisión de literatura. El objetivo de esta guía fué para rescatar las preguntas centrales del libro Summing up de Light y Pillemer (1984), y aplicarlas al tema de revisión seleccionado. 
B) Una vez que se explicitó la pregunta a contestar, se tomaron como criterios de inclusión para la revisión, todos los documentos posibles, publicados y no publicados, que abordaran el planteamiento de modelos explicativos sobre las determinantes y condicionantes del proceso salud-enfermedad.

C) Determinados los criterios de inclusión se hizo la consulta en el sistema computarizado de dos programas de referencias bibliográficas: MEDLINE y LILACS. En esta revisión se dieron como palabras-clave al sistema de búsqueda de bibliografía las siguientes: modelos explicativos, determinantes, salud-enfermedad.

D) Una vez que el programa de cómputo hizo las posibles combinaciones de las palabras-clave, se registraron 80 artículos de los cuales se obtuvo un resumen de cada uno para ser estudiado y decidir la pertinencia de incluirlo en la revisión.

E) Del resultado de la revisión inicial, sólo 17 artículos abordaban cualitativa o cuantitativamente el análisis sobre las condicionantes y determinantes del fenómeno de estudio. Los sesenta y tres artículos restantes fueron descartados para continuar la revisión debido a que sus planteamientos no eran pertinentes para el estudio exploratorio que se planteó desarrollar. F) Además de los 17 artículos publicados se encontraron dos artículos no publicados que abordan el análisis sobre las determinantes y condicionantes del fenómeno de estudio. Por lo tanto, los artículos para la revisión del ensayo comprendió un total de 19; de estos 13 fueron básicamente cualitativos y 6 cuantitativos.

G) Para hacer un estudio comparativo y confrontar los modelos encontrados, se determinó delimitar para cada propuesta, las variables incluídas en cada modelo, hipótesis de trabajo del modelo, ventajas, desventajas, época en que surge y representantes; todo ello con el fin de poder hacer un análisis comparativo de la metodología de cada modelo y del conflicto de los diferentes hallazgos. El hecho de delimitar los puntos mencionados para cada modelo, surgió de la necesidad de discutir hasta que punto la metodología de análisis, los planteamientos fundamentales y los aportes de cada modelo son compatibles, complementarios, contradictorios, científicamente sólidos, tendenciosos, conservadores, críticos y/o reflexivos.
H) Una vez que se delimitaron los puntos correspondientes a cada modelo, se procedió a hacer la discusión y conclusiones de la revisión. En este caso la discusión se centró sobre los siguientes puntos:

- Metodologías de abordaje al problema - tipo de estudio;

- Hipótesis de trabajo y variables de análisis incluidas;

- Variación de resultados y hallazgos en conflicto.

\section{MODELOS TEORICOS DEL PROCESO SALUD-ENFERMEDAD}

El análisis de modelos teóricos que abordan como mismo objeto de estudio y desde diferentes disciplinas el proceso salud-enfermedad, incluyó once propuestas. Es necesario aclarar que no se trata de describir cada modelo, sino que para fines de análisis se trató de delimitar las hipótesis de trabajo, variables, ventajas, desventajas, época y representantes de cada modelo, mismas que se plantean explícitamente a continuación.

\section{Modelo Mágico-Religioso}

Para esta propuesta, la enfermedad resulta de fuerzas ó espíritus; representa un castigo divino, o bien se trata de un estado de purificación que pone a prueba la fé religiosa. Las fuerzas desconocidas y los espíritus (benignos y malignos) constituyen las variables determinantes $y$ condicionantes del estado de salud-enfermedad que priva en un individuo ó en una sociedad. Este modelo facilita la aceptación de la muerte inminente pero también circunscribe la prevención a la obediencia de normas y tabúes, y la curación a la ejecución de ritos. Su principal desventaja es que impide el avance cognoscitivo a la vez que fomenta la actividad pasivo-receptiva del hombre. Como seguidores de este modelo podemos nombrar a las sociedades primitivas, desde la edad media hasta la actualidad, teniendo como representantes a chamanes, brujos, curanderos, sacerdotes y espiritistas (Piña, 1990). 


\section{Modelo Sanitarista}

Para este modelo, la salud-enfermedad es consecuencia de las condiciones insalubres que rodean al hombre, en este sentido, las condiciones ambientales son los determinantes primarios, promoviendo la introducción de medidas de saneamiento contra los índices de morbimortalidad. La principal limitante de este modelo, es el hecho de que no contempla los factores sociales que determinan la prevalencia de condiciones de vida insalubres para las diferentes clases sociales. Su época y representantes lo constituyen la revolución industrial europea con la penetración capitalista en las colonias, $y$ Smith y Pettenkofer, respectivamente (Piña, 1990).

\section{Modelo Social}

El elemento central de análisis que propone este modelo, es que la salud-enfermedad se genera en las condiciones de trabajo y de vida del hombre y de cada conjunto poblacional. Introduce como variables determinantes el estilo de vida, factores del agente y factores del ambiente. Privilegia a lo social como el factor más importante, factor que explica la aparición y el rol de otros factores participantes. Su problema fundamental es que en su aplicación como herramienta de análisis, se corre el riesgo de reducir la complejidad real del proceso salud-enfermedad a la problemática de las relaciones sociales. Se puede considerar que los máximos representantes de este modelo son: Peter Frank, Virchow y Ramazzini (siglo XIX) (Piña, 1990).

\section{Modelo Unicausal}

La salud-enfermedad es la respuesta a la presencia activa de agentes externos. Constituye un fenómeno dependiente de un agente biológico causal y de la respuesta del individuo, donde se busca el agente patógeno ó el factor causal de cada enfermedad. Este modelo permitió la investigación de medidas de control y de fármacos que revolucionaron el tratamiento individual del enfermo; no obstante no explica porqué el mismo agente no produce siempre enfermedad por lo que descifra de manera parcial las causas de la enfermedad sin aclarar el rol de otros factores. El surgimiento de este modelo se dió en la segunda mitad del siglo XIX y a principios del XX, teniendo como máximos representantes a Pasteur y a Koch (Pasteur, 1946).

\section{Modelo Multicausal}

La influencia simultánea de factores que corresponden al agente, al huesped y al ambiente, son en primera instancia los condicionantes del estado de salud que guarda el individuo ó un conjunto poblacional. A través del análisis de las variables que incluye este modelo se pueden conocer más de un factor participante en el fenómeno de estudio, sobre los cuáles se puede actuar preventivamente. Como principal desventaja, aparece el hecho de que no establece el peso específico de cada factor y continúa un énfasis sobre lo biológico e individual, mientras que lo social aparece incluído en el entorno. La segunda mitad del siglo XX (50s) fué la época de aparición de esta propuesta $y$ sus representantes pudieran ser Leavell y Clark (Leavell \& Clark, 1953; Noble, 1988).

\section{Modelo Epidemiológico}

Incorpora el modelo multicausal para el estudio de la salud-enfermedad colectiva e introduce la red de causalidad, donde el elemento central de análisis es la identificación de los factores de riesgo, elemento que constituye su mayor ventaja. Su limitación fundamental es de acuerdo a esta propuesta, el valor de cada factor de riesgo depende de la distancia y del tamaño del efecto en la red de causalidad, además de que lo biológico y lo social aparecen como factores indiferenciables. Este modelo se generó en la segunda mitad del siglo $\mathrm{XX}(60 \mathrm{~s})$ y sus representantes fueron: MacMahon $y$ Pugh (1975).

\section{Modelo Ecológico}

Para el modelo ecológico, teniendo como principal representante a Susser (70s), la salud-enfermedad resulta de la interacción agente-huésped-ambiente en un contexto tridimensional que descubre tanto las relaciones de factores causales entre sí, como las relaciones directas 
con el efecto. Si bien es cierto, este modelo retoma el análisis de las mismas variables que incluye el modelo multicausal, también es cierto que su abordaje permite asignar un valor específico a cada factor involucrado en el proceso de estudio. Esta propuesta no explica la génesis de perfiles diferenciales de salud-enfermedad ya que carece de conceptos y métodos adecuados para abordar lo social (Susser, 1972).

\section{Modelo Histórico-Social}

Desde la perspectiva de esta propuesta, existen perfiles diferenciales de salud-enfermedad que guardan una estrecha relación con el contexto histórico, el modo de producción y las clases sociales. Todos los factores causales se permean por lo social-histórico. Introduce cinco variables fundamentales para el análisis del objeto de estudio: la dimensión histórica, la clase social, el desgaste laboral del individuo, la reproducción de la fuerza de trabajo y la producción del individuo. Su aporte especial es que incorpora la dimensión histórica-social al análisis epidemiológico, a la vez que aporta nuevas categorías de análisis y cuestiona la eficacia de la prevención y control de la salud-enfermedad manteniendo intactas las relaciones de explotación que la generan. Al igual que el modelo social, en su aplicación existe el riesgo de reducir la complejidad real a la problemática de las relaciones sociales y de la dimensión histórica. Sus representantes son: Berlinguer, Laurell y Breilh (70s) (Breilh \& Grand, 1988; Laurell, 1985; Bloch, 1985; Laurell \& Noriega, 1987; Martins et al., 1987; Ciari \& Siqueira, 1976).

\section{Modelo Geográfico}

La enfermedad resulta de la interacción de factores patológicos y factores propios del ambiente geográfico (factores geógenos); privilegia el ambiente geográfico como factor determinante del proceso salud-enfermedad. Aunque contempla el factor social como parte de los factores geógenos no da la suficiente importancia como determinante del proceso. Este modelo se generó en el presente siglo (50s) y sus principales representantes son: Jaques May y Voronov (May, 1977).

\section{Modelo Económico}

Incorpora la teoría del capital humano en los determinantes de la salud enfermedad y conceptualiza a la salud como un bien de inversión y de consumo para estar alerta ante la enfermedad. Para esta propuesta el ingreso económico, los patrones de consumo, los estilos de vida, el nivel educativo y los riesgos ocupacionales son las variables que entran en juego en el análisis de los determinantes de la salud y la enfermedad. Incorpora y justifica el valor económico en el estudio del proceso en cuestión y da una visión mas amplia del determinante social. Bajo esta perspectiva existe el riesgo de tomar una posición reduccionista hacia lo económico, ya que se plantea un exceso de racionalidad en el análisis de los determinantes. Esta propuesta de análisis es paralela al surgimiento de la economía de la salud en años recientes (1970-80) y sus principales representantes son Anne Mills, Gilson y Muskin (Mills \& Gilson, 1988; Muskin, 1962).

\section{Modelo Interdisciplinario}

El estado de salud-enfermedad, tanto a nivel individual como social, resulta de la interacción de factores que se abordan de manera interdisciplinaria y que operan jerárquicamente en diferentes niveles de determinación. Existen determinantes básicos a nivel sistémico (ambiente, genoma, etc.), determinantes estructurales a nivel socio-estructural (estratificación social, mecanismo de redistribución de la riqueza, etc.), determinantes próximos a nivel institucional-familiar (estilos de vida, sistemas de salud, etc.) y, a nivel individual el propio estado de salud. La principal ventaja de esta propuesta es que intenta proponer un enfoque integral para el estudio de los determinantes del proceso de estudio (factores demográficos, epidemiológicos, económicos, sociales, políticos, etc.); su principal desventaja es que al igual que otros modelos, no desagrega la influencia de los factores que considera y por lo tanto parece ser que no pondera el valor específico de cada determinante. Este modelo surgió a principio de los 90 s y sus representantes son Julio Frenk y col. (Frenk et al., 1991; Arredondo, 1992). 
Es importante indicar que de la revisión de los 19 documentos seleccionados, los 5 de carácter cuantitativo se refieren fundamentalmente al modelo social-histórico justificando el modelo con hechos empíricos cuantificables, mientras que para el resto de los modelos sólo fueron de carácter cualitativo, donde se da por entendido que sus planteamientos teóricos se derivan de hallazgos de otros autores que no se atrevieron a plantear un modelo concreto.

\section{DISCUSION Y CONCLUSIONES}

En relación a las metodologías de abordaje al problema y tipo de estudio que permitió llegar a construír un modelo teórico, se pueden comentar los siguientes puntos:

Cada modelo tiene una hipótesis implicita ó explícita en relación a los determinantes del proceso salud-enfermedad y a excepción de los artículos referidos sobre el modelo históricosocial, en los modelos restantes se limitan a aspectos cualitativos que derivan de hallazgos ó experiencias anteriores y donde no se mencionan los tipos de diseño y metodología de estudio que conducieron a tales hallazgos, no obstante se deja entender que los hallazgos que retoman son "válidos" de no ser así el modelo que plantean no tendría "validez".

Otro aspecto a resaltar es que no es claro poder entender si las hipótesis que los conducen son verificables y/ó falsables. Al parecer cada autor ó representante del modelo está interesado en verificar su planteamiento, pero ningún autor se ha planteado la necesidad de falsar sus propuestas teóricas. Si ellos consideran que construir el conocimiento es verificar, creo que más que construirlo lo están limitando pues el hecho de no abordar la falsabilidad de los enunciados teóricos es una gran limitante para el progreso del conocimiento.

Aquí vale la pena recordar a Popper, al referirse que la ciencia no tienc nada de absoluta y que la estructura de sus teorías se eleva sobre un terreno pantanoso. Al parecer los representantes de los modelos olvidan lo anterior y más bien se preocupan por legitimar sus modelos y darles un sentido paradigmático, hablando en palabras Kuhnianas, mismo que los haga afirmativos, verificables, "científicamente aceptables" y por supuesto irrefutables; como es común, es posible que se considere erróneamente que lo irrefutable es la mejor manera de construír el conocimiento, por lo tanto dificilmente se da válidez al hecho de la falsabilidad de los enunciados hipotéticos.

Luego entonces, no es fácil entender si las confirmaciones ó verificaciones que se hacen son efectivamente el resultado de predicciones riesgosas, es decir que no sólo se basen en la teoría explícita de cada modelo, sino que se puedan confrontar a otras teorías, de manera que se produzcan hallazgos incompatibles y de refutación. Al respecto, hay que considerar que todo modelo teórico que no es refutable por ningún suceso concebible no es científico, por lo que la irrefutabilidad no es virtud de una teoría (como comunmente se cree), sino un vicio.

En relación a las variables de análisis incluídas en cada modelo, es interesante observar que cada autor incluye variables complementarias o de continuidad cronológica a los modelos anteriores a su época de acuerdo al avance de los paradigmas "científicos" por lo que no es tan casual que el modelo "interdisciplinario" incluya a todas las variables que se plantearon en modelos que le precedieron. Hay que señalar también que sólo dos modelos resaltan al incluir variables determinantes que desequilibran a los modelos restantes: el mágico-religioso y el histórico-social.

En relación a la variación de resultados y hallazgos en conflicto, para fines de este ensayo que más bien es de carácter exploratorio y no de prueba de hipótesis, se debe aclarar que la discusión se abordó mediante el análisis de las variables incluídas y no propiamente sobre los hallazgos.

En relación a los modelos sanitarista, unicausal, multicausal, epidemiológico, ecológico, social, geográfico, económico e interdisciplinario, no hay conflictos aparentes internodelos, más bien se observa que las variables incluidas son complementarias y no salen de cierto juego "científico" con tendencias muy claras a validar una práctica dirigida a que prevalezca el status quo. Si existe algún conflicto, este se dá en la manera que cada autor enfoca su análisis y en el peso que se asigna a cada variahle deterninante del modelo. Incluso me atrevería a decir 
que hay modelos que además de no entrar en conflicto son demasiado repetitivos, tal es el caso de los modelos multicausal y ecológico, donde sus variables de análisis macro son idénticas.

Por otra parte, el conflicto intermodelos si aparece cuando confrontamos las variables de los modelos incluídos en el párrafo anterior con las de los modelos mágico-religioso e históricosocial. Es decir que cuando hablamos de "fuerzas desconocidas", "espíritus", dimensión histórica, clase social, desgaste, reproducción de la fuerza de trabajo y producción del individuo como determinantes explicativos del proceso Salud-Enfermedad, aparece un serio conflicto con las determinantes de los otros modelos.

Finalmente, además de todo lo dicho, es necesario resaltar las siguientes conclusiones que den el cierre al presente documento:

$\mathrm{Si}$ bien es cierto el desarrollo de estos modelos ha dado avances importantes en el entendimiento de los determinantes y condicionantes del proceso salud-enfermedad, también es cierto que es cuestionable hasta que punto los modelos revisados contienen lo que comunmente se denomina "sesgo convencional", es decir sí en algún momento, en el caso de que algunas teorías propuestas fueron genuinamente testables, al encontrarse falsas siguen contando con el sostén de sus "admiradores", induciendo algún supuesto auxiliar ad hoc ó reinterpretando la teoría de manera que escape a su refutación.

El conflicto de hallazgos-variables intermodelos que se discutió en esta sección, causa un desequilibrio de gran importancia en la construcción de teorías, por lo que debe ser el punto de partida para abordar el desarrollo de un modelo teórico-integral-operativo, que permita abatir la fragmentación del conocimiento observada en la confrontación de los modelos revisados y que replantee la explicación de los determinantes y condicionantes del fenómeno de estudio; después de todo, como Piaget lo aprecia, la construcción, avance y progreso del conocimiento debe surgir de los desequilibrios y conflictos que se dan en cada fase de su desarrollo.

Las consecuencias del problema analizado sobre la formación de recursos humanos en salud son de considerarse, si tomamos en cuenta que estos modelos no son del todo acabados, y más aún por el hecho de que son un elemento importante en el diseño de los programas educativos para la formación de personal en salud.

Llevar a los programas educativos de formación de recursos humanos en salud un conocimiento que se desarrolla en una arena de lucha hegemónica del pensamiento, y donde no sólo cada propuesta pretende dejar su huella intelectual, sino que además, algunas veces, tratan de subordinar ó marginar a otras teorías, tiene como consecuencia que el conocimiento teórico del proceso salud-enfermedad no pueda interactuar con la práctica del fenómeno; esto último es algo que deberán tener presente los líderes de la formación de personal de salud, después de todo la tarea esencial de dicho personal es llevar a la práctica lo aprendido en la academia.

Tomando en cuenta lo anterior, así como el análisis y reflexión que se hizo en este ensayo, quedan como punto de reflexión los siguientes cuestionamientos:

¿. Los contenidos teórico-metodológicos de programas de formación, son realmente significativos para dar respuesta a las condiciones de salud-enfermedad que privan en los diferentes conjuntos poblacionales ?

¿Los modelos explicativos que se han desarrollado hasta que punto ayudan u obstaculizan, tanto el avance del conocimiento sobre los determinantes y condicionantes del proceso salud-enfermedad como el entendimiento $y$ forma en que se da la respuesta social organiza$\mathrm{da}$ ante los retos que plantean las necesidades de salud de cada grupo social?

\section{RESUMO}

\section{ARREDONDO, A. Análisis y Reflexión} sobre Modelos Teóricos del Proceso SaludEnfermedad. Cad. Saúde Públ., Rio de Janeiro, 8 (3): 254-261, jul/set, 1992.

Este trabajo parte de la necesidad de hacer una revisión y análisis de los diferentes modelos teóricos sobre los determinantes y condicionantes del proceso salud-enfermedad como herramienta relevante de los programas educativos en la formación de recursos humanos. Determinando para cada modelo la 
hipótesis propuesta, las variables de estudio, ventajas, desventajas, época y representantes, se plantea como objeto de análisis el carácter científico y/ó metodológico de los modelos: mágico-religioso, sanitarista, unicausal, multicausal, epidemiológico, ecológico, social, histórico-social, geográfico, económico e interdisciplinario. La discusión y conclusiones se centran en la necesidad de discutir hasta que punto la metodología, los planteamientos $y$ aportes fundamentales de cada modelo son compatibles, complementarios, contradictorios, científicamente sólidos, tendenciosos, conservadores, críticos y reflexivos, asi como su relevancia en la formación de recursos humanos en salud.

Palavras-Chave: Salud-Enfermedad; Determinantes; Modelos Teóricos

\section{REFERÊNCIAS BIBLIOGRÁFICAS}

ARREDONDO, A., 1992. Aportes de las ciencias sociales para el análisis de la morbi-mortalidad. Revista Mexicana de Educación Médica (en dictámen).

BLOCH, C., 1985. El proceso salud-enfermedad en el primer año de vida. Estudio de una cohorte, Rosario (Argentina), 1981-1982. (Primera parte). Cuadernos Médico Sociales, 32: 5-19.

BREILH, J. \& GRANDA, E., 1988. Investigación en salud en la sociedad: guia pedagógica sobre un nuevo enfoque del método epidemiológico. Quito: Ediciones C.E.A.S.

CIARI, J. \& SIQUEIRA, A., 1976. Problemas de saúde materno-infantil no Brasil. Saúde da comunidade: Temas de medicina preventiva e social. Rio de Janeiro: McGraw-Hill do Brasil.

FRENK, J., 1991 Elements for a theory of the Health Transition. (Mimeo.)

LAURELL, C., 1985. El estudio social del proceso salud-enfermedad en América Latina. Ponencia presentada en el coloquio "La Santé en Amerique Latine, realité socio-politique et projects des soins primaires". Montréal: AMALC.
LAURELL, C. \& NORIEGA, M., 1987. Proceso de trabajo y salud en Sicartsa. Cuadernos Médicos Sociales, 40: $25 \cdot 47$.

LEAVELL, H. \& CLARK, E.G., 1953. Epidemiology and Multicausal. Textbook of Preventive Medicine. New York: McGraw Hill.

LIGHT, R. \& PILLEMER, D., 1984. Summing Up: The Science of Reviewing Research. Boston: Harvard College.

MacMAHON, B. \& PUGH, T., 1975. Principios y Métodos de Epidemiología. 2" ed., México, D.F.: Prensa Médica Mexicana.

MARTINS, J.; ALVARENGA, A. T.; SIQUEIRA, A. A. F.; SZARJARA, S. C. \& LIMA, F. D., 1987. As determinaçōes biológica e social da doença: Um estudo de anemia ferropriva. Revista de Saúde Pública, 21: 73-89.

MAY, J., 1977. Medical Geography: its methods and objetives. Social Science and Medicine, 5: 715730.

MILLS, A. \& GILSON, L., 1988. Health Economics for Developing Countries: A survival kit. England: EPC publication No. 17.

MUSHKIN, S., 1962. Health as an investment. Journal of Political Economy, 2: 129-157.

NOBLE, S., 1988. A Multicausal Solution? Hidden Arguments/New Brunswik: Rutgers University Press.

PASTEUR, L., 1946. Memorias de Luis Pasteur. Conmemoración del bicentenario de la muerte de Pasteur. México, D.F.: Sociedad Mexicana de Historia Natural.

PIÑA, B., 1990. Modelos de explicación sobre la determinación del proceso Salud-Enfermedad. Factores de riesgo en la comunidad; Tomo I. México. D. F.: Universidad Autonoma de Méxjco.

SUSSER, M., 1972. Agent, Host and Enviroment as an ecological system. Causal Thinking in the Health Sciences. New York: Oxford University Press.

TERRIS, M., 1975. Approaches to an epidemiology of health. American Journal of Public Health. 65: 1037-1045. 